\title{
MONITORING OF A PRESTRESSED BRIDGE MODEL BY ULTRASONIC MEASUREMENT AND VIBRATION RECORDINGS
}

\section{Chun-Man Liao, Falk Hille, Daniel Fontoura Barroso and Ernst Niederleithinger}

Bundesanstalt für Materialforschung und -prüfung (BAM), Unter den Eichen 87, 12205 Berlin, GERMANY

\{Chun-Man.Liao, Falk.Hille, Daniel.Barroso, Ernst.Niederleithinger\}@ bam.de

\begin{abstract}
The aim of this work is to improve the current structural health monitoring (SHM) methods for civil structures. A field experiment was carried out on a two-span bridge with a built-in unbonded prestressing system. The bridge is a 24-metre long concrete beam resting on three bearings. Cracks were formed subsequently when a prestressing force of $350 \mathrm{kN}$ was changed to $200 \mathrm{kN}$, so that different structural states could be demonstrated. The structural assessment of this reference bridge was accomplished by the non-destructive testing using ultrasonic devices and vibration measurements. The ultrasonic velocity variations were investigated by using the coda wave interferometry method. The seismic interferometry technique was applied to the vibration recordings to reconstruct the wave propagation field in the bridge. This investigation shows that the wave velocity is sensitive to the current structural state and can be considered as the damage indicator. Overall, the implementation of coda cave interferometry and seismic interferometry technique facilitates structural health monitoring (SHM) in civil engineering.
\end{abstract}

Keywords: structural health monitoring, non-destructive testing, coda wave interferometry, seismic interferometry, ultrasonic measurement, prestress. 


\section{INTRODUCTION}

The application of prestressing and post-tensioning techniques to civil infrastructures such as long span bridges improves the structural performance in their lifetime. Since the prestress loss can occur while the structure is in service, it is important to monitor the prestressed civil structures. However, a lot of studies lack the reference condition of large-scale test objects for comparison in the evaluation.

In our study, a large-scale concrete bridge model containing a built-in unbonded prestressing system was monitored. The variable prestressing force was a benefit to our investigation on the bridge. As the structural capacity was enhanced by the high prestressing force, the structural condition was considered as the baseline for damage detection. Thus, we could explore damage indicators of the prestressed bridge.

To achieve the research aim, both active and passive procedures were applied in the field experiment. Since damage leads to changes in the dynamic properties of the structure, vibration monitoring is useful as a non-destructive evaluation tool. However, the measured eigenfrequencies are also sensitive to environmental influences [1]. Referring to the advantage of the wavebased method, two kinds of wave propagation were considered in our study: ultrasonic wave propagation, which is widely used for non-destructive testing (NDT), and the resultant of seismic interferometry of noise recordings.

Ultrasonic waves are sensitive to variations of several parameters (stress, temperature, moisture) and to any kind of damage. Different mechanical wave propagation regimes have been investigated for concrete so far [2]. In the attenuation phenomenon, the waves scatter and induce the onset of late arrival, constituting the so-called ultrasonic coda. In seismology, the coda waves are known for its high sensitivity to small perturbations of the medium. Coda waves are defined as the part of the signal that occurs directly after the arriving phases. In a heterogeneous medium, the small change has less influence on the waves arriving first than on the diffusion waves arriving later. As coda waves travel longer distances, the sampling repeats and the influence of the small change is amplified [3]. Hence, the coda energy change is more detectable than the direct ones.

Recently, coda wave interferometry (CWI) has been used to ultrasonic coda and noise recordings in civil structures. The implementation of CWI enabled the detection of early subtle changes in reinforced concrete structures until crack formation [4]. In an earlier study [5] using ultrasound to monitor stress distribution and cracking in concrete beams, they were loaded until failure to detect cracks before visual signs appear on the surface. The application of CWI to the waveform of the cross-correlation function of two vibration records was presented in another study [6] to analyse the wave velocity variation.

This contribution introduces the background of CWI and the application of CWI to our measurement data. We studied the effect of the post-tensioning force change based on the coda wave velocity variation. The experiment result confirmes the applicability of the monitoring method using CWI. This research shows the progress towards the development of a cost-effective and reliable technique for real-time monitoring in large-scale structures.

\section{CODA WAVE INTERFEROMETRY}

We studied the effect of the post-tensioning force change based on the coda wave velocity variation. For this, the same waveform in two different bridge states was compared using coda wave interferometry (CWI). The principle of CWI is to compare the waveform consistency of coda waves recorded in two different states. Figure 1 shows two signals recorded before and after perturbation. The first arrivals of the signals are almost the same, while the coda wave 
(later arrivals) shows significant wave propagation time lags. The relative time lag $\left(\frac{\delta t}{t}\right)$ between the two signals before and after the perturbation indicates the relative wave velocity change $\left(\frac{\delta v}{v}\right)$. Since the prestress has a homogeneous effect on the wave propagation velocity, the linear relation is applied

$$
\frac{\delta v}{v}=-\frac{\delta t}{t}
$$

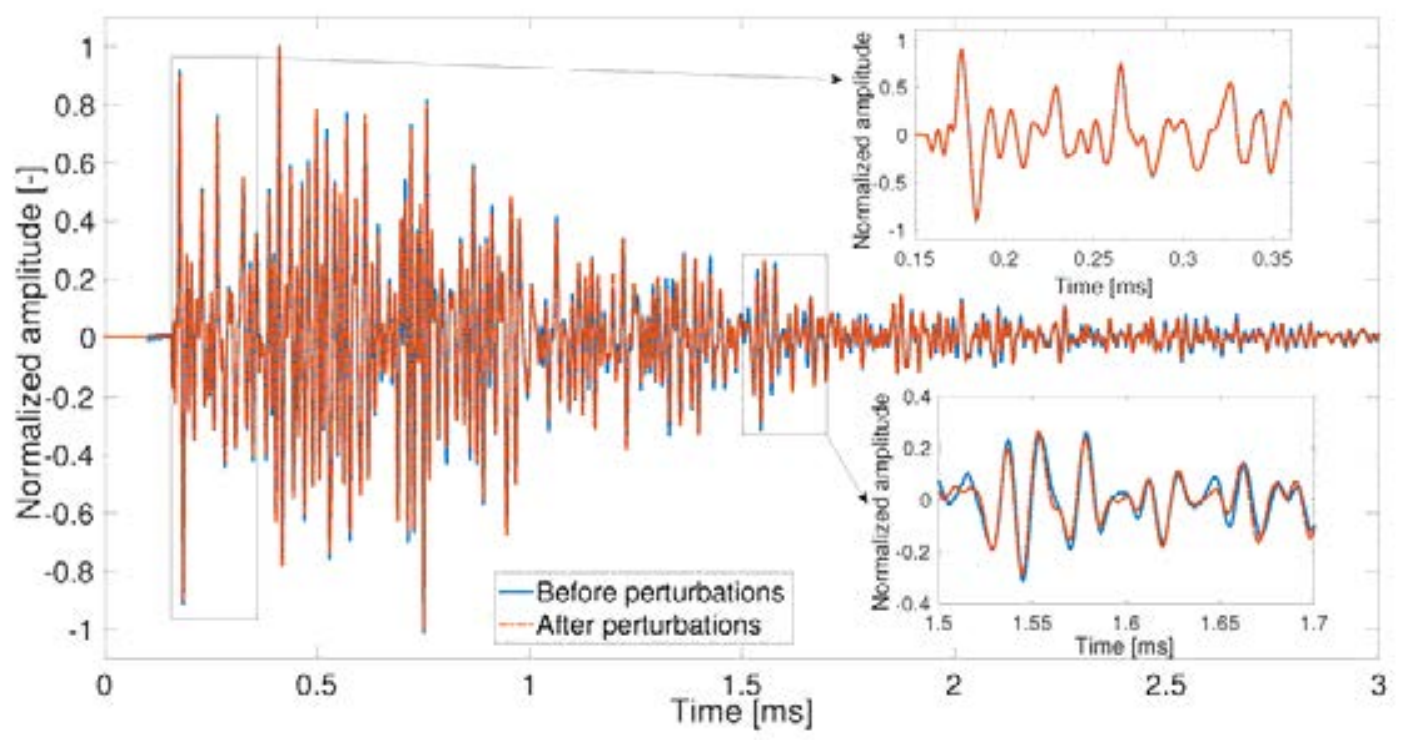

Figure 1: Repeated ultrasonic signals with difference just visible in the coda [7].

The following explains how to estimate the wave velocity variation by applying the stretching method to CWI.

\subsection{The stretching method}

As the relative velocity decrease is defined as the stretch factor $\alpha$

$$
\alpha=\frac{\delta v}{v}
$$

the shift time in wave propagation is represented by $t(1+\alpha)$.

Hence:

$$
u_{\mathrm{s}}=u_{\mathrm{o}}(t(1+\alpha))
$$

where the original waveform $u_{\mathrm{o}}$ is stretched and turned into $u_{\mathrm{s}}$.

According to this formulation, the sign of $\alpha$ is negative if the waveform is compressed. lows

The cross-correlation between the stretched and the original waveform is then shown as fol-

$$
C C(\alpha)=\frac{\int_{t-T}^{t+T} u_{o}\left(t^{\prime}\right) u_{s}\left(t^{\prime}\right) d t^{\prime}}{\sqrt{\int_{t-T}^{t+T} u_{o}^{2}\left(t^{\prime}\right) d t^{\prime} \int_{t-T}^{t+T} u_{s}^{2}\left(t^{\prime}\right) d t^{\prime}}}
$$

The cross-correlation coefficient (CC) is considered as a quality indicator. If two waveforms are identical, the cross-correlation coefficient is equal to 1. Vice versa, the cross-correlation coefficient between -1 and less than 1 indicates the difference depending on the dilation level. 


\subsection{Application}

We investigated the impulse response in the different prestressed states using coda wave interferometry. One waveform was considered as a reference and the other was stretched by the factor $\alpha$ in a range $[-d v, d v]$. The best $\alpha$ was determined by comparing CWI in the reference waveform and the stretched waveform. Such an $\alpha$ maximizes the cross-correlation of two compared waveforms. At the same time, the wave velocity change is obtained in the best fit condition. As a result, we could study the relationship between the prestressing force variations, temperature change and wave propagation velocity in the concrete bridge model.

\section{EXPERIMENT}

\subsection{Test structure BLEIB}

The test structure was a prestressed concrete bridge model exposed to the nature environment at the BAM test site [8]. No traffic vibrations were induced as background noise on the bridge. The bridge model has been used for various loading and vibration tests. Thereby, vertical cracks had developed near the two midspans (Fig. 2). For our study, no additional external load was applied to the bridge, thus no significant post-cracking behavior occurred. This reduced the confusion of the external interferon.

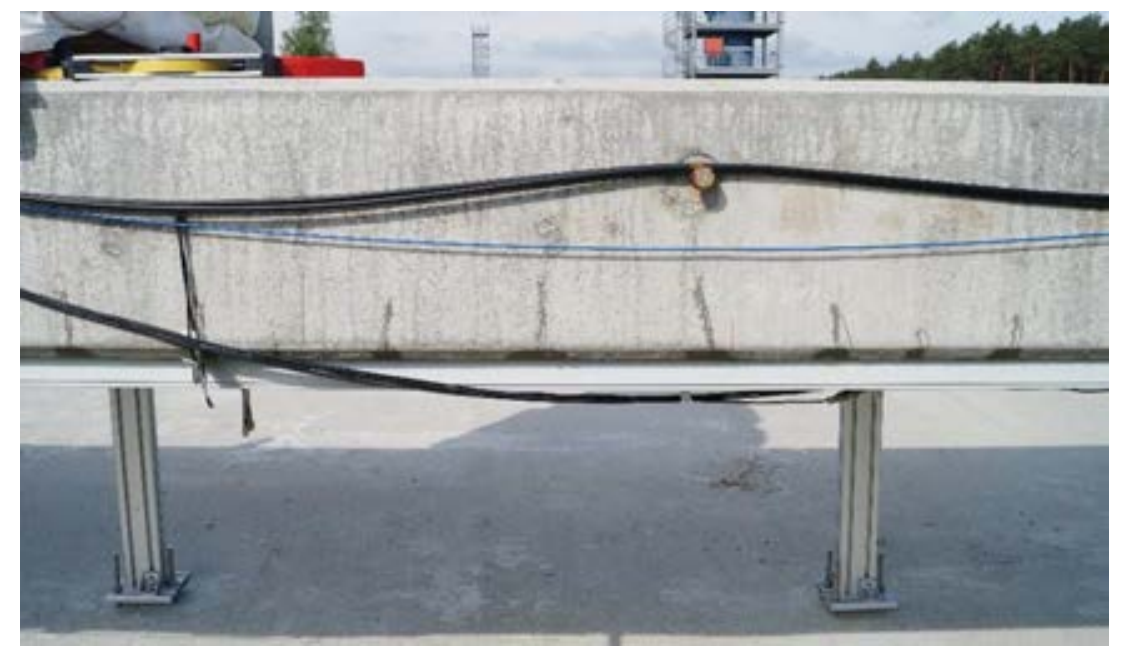

Figure 2: The damage condition after vibration and loading tests (photographed in 2018).

We induced the natural damage by opening the already existing cracks. Initially, we increased the prestressing force up to $550 \mathrm{kN}$ (level 1 in Tab. 1) and then adjusted the magnitude of the prestressing force (level 2-9 in Tab. 1) to obtain the different event extensions of the already existing cracks.

\begin{tabular}{ll}
\hline Level & $\begin{array}{l}\text { Prestressing } \\
\text { force }[\mathrm{kN}]\end{array}$ \\
\hline 1 & 553 \\
2 & 457 \\
3 & 403 \\
4 & 353 \\
5 & 300 \\
6 & 255
\end{tabular}




\begin{tabular}{ll}
7 & 200 \\
8 & 353 \\
9 & 455 \\
\hline
\end{tabular}

Table 1: Prestressing force level.

\subsection{Vibration measurements}

We implemented passive monitoring by vibration measurement. Geophones (Fig. 3 left) were used and the sampling rate was $600 \mathrm{~Hz}$. The blue markers in Fig. 4 indicate the geophones' position. The distance between every two adjacent geophones is $3 \mathrm{~m}$.
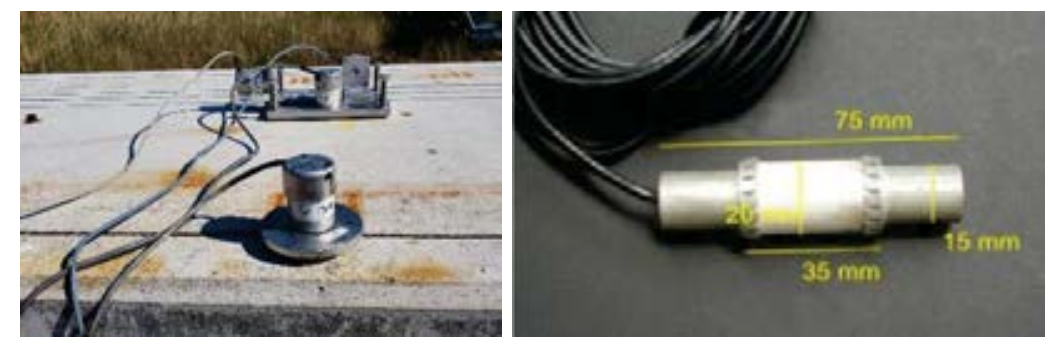

Figure 3: Sensors. Left: geophones. Right: ultrasonic transducers SO807 [9].

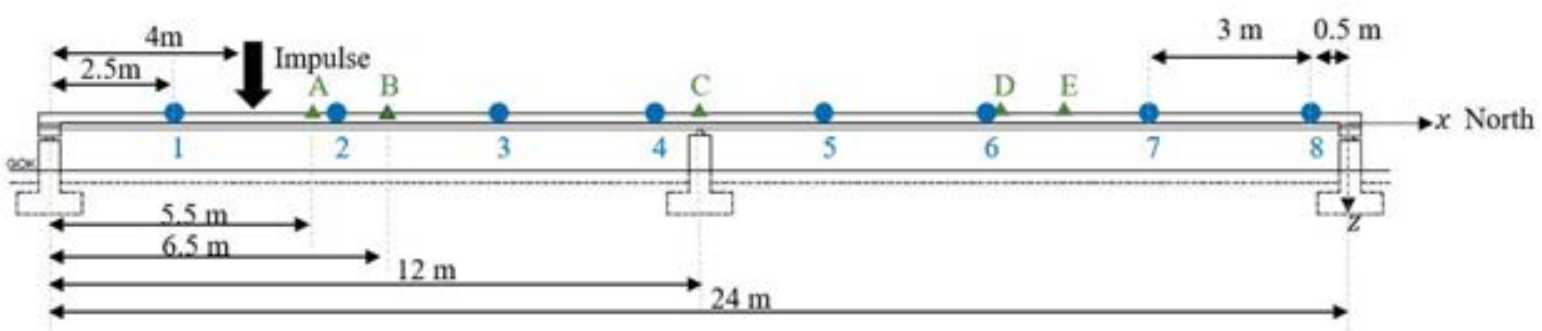

Figure 4: Side view of the bridge and sensor positions.
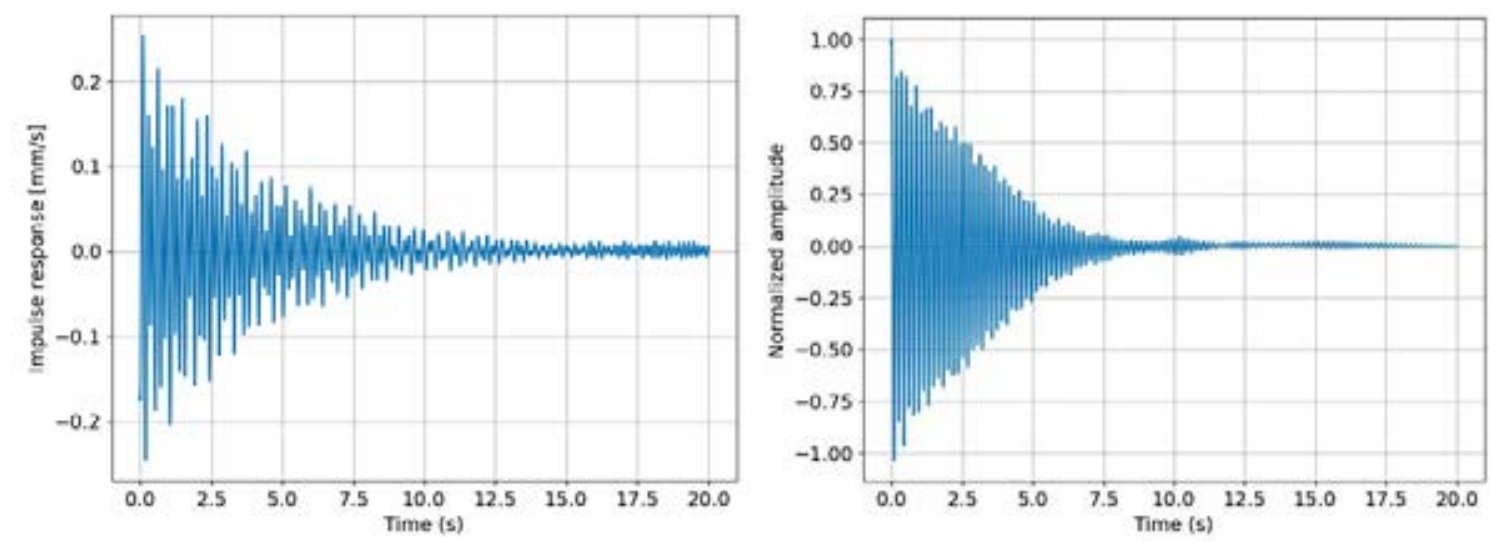

Figure 5: Impulse response in Level 2. Left: hammer impact received at the position 2. Right: seismic interferometry of the ambient noise at the position 1 and 2 .

We directly obtained the real impulse response caused by the impulse source excited by the hammer impact (the position reference to the black arrow in Fig. 4). The measured impulse response lasted $20 \mathrm{sec}$. Fig. 5 on the left shows the impulse response received at the position 2. We also performed the ambient vibration measurement for $3 \mathrm{~min}$. for each prestressing level. To extract the virtual impulse response, the seismic interferometry technique (the cross- 
correlation method) dealing with the reconstruction of Green's functions between two receivers was used. One of these two receivers was turned into a virtual source. Finally, two virtual impulse responses extracted from 3-minute measurement data were stacked and normalized. Fig. 5 on the right shows the impulse waveform with the window size of $20 \mathrm{sec}$. between position 1 and position 2 .

\subsection{Ultrasonic measurements}

For active monitoring, we performed the ultrasonic transmission measurement. 14 ultrasonic transducers "SO807" ([9], Fig. 3 right) were mounted inside the bridge during its construction before casting (position reference to the green markers in Fig. 4 and Fig. 6). This sensor can operate as both a transmitter and a receiver. The ultrasonic wave was generated with a central frequency of $60 \mathrm{kHz}$. The sampling rate is $1 \mathrm{MHz}$. In the measurement, 10000 samples were taken.

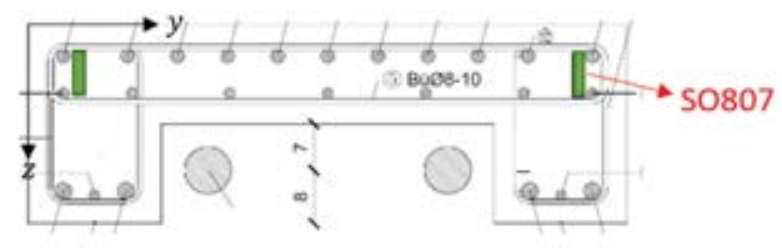

Figure 6: The embedded SO807 sensors in a cross section.

\section{RESULTS}

First, we show the modal analysis results of the bridge finite element model and the evaluation of the influence of the prestressing force on the natural frequency. Then the results of the ultrasonic impulse response, the real impulse response and the virtual impulse response are presented. The influence of temperature and prestressing on the coda waves is finally discussed.

\subsection{Natural frequency and structural stiffness}

The opening/close of the already existing cracks caused the overall stiffness change. Consequently, the structural dynamic behaviour changed due to the superposition of two effects from the prestressing force and the cracks. The comparison of the first natural frequency in the simulation of the intact bridge model (Fig. 7) with the measured result in the Level 1 state (Fig. 8 left) showed that the current bridge condition is damaged.
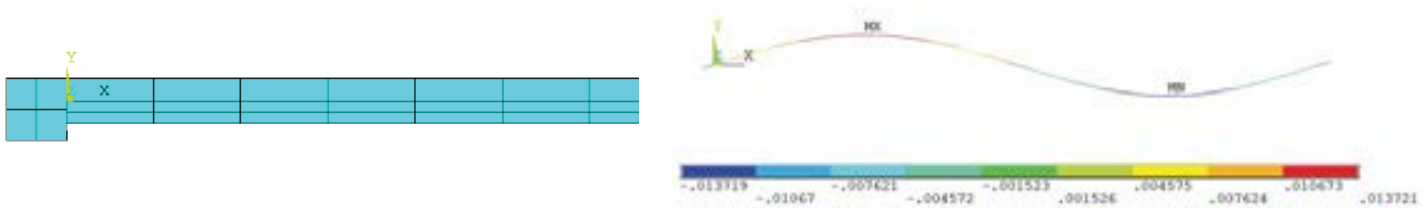

Figure 7: Half span of the finite element (FE) model (left). The first mode shape corresponds to $3.9 \mathrm{~Hz}$ (right).

Furthermore, Fig. 8 shows the first two modal frequencies in the Level 1 and 7 states. The decrease of natural frequencies depends on the prestress loss and the event extension of the already existing cracks in the bridge. Based on this, we were able to demonstrate different structural states by adjusting the prestressing force. 

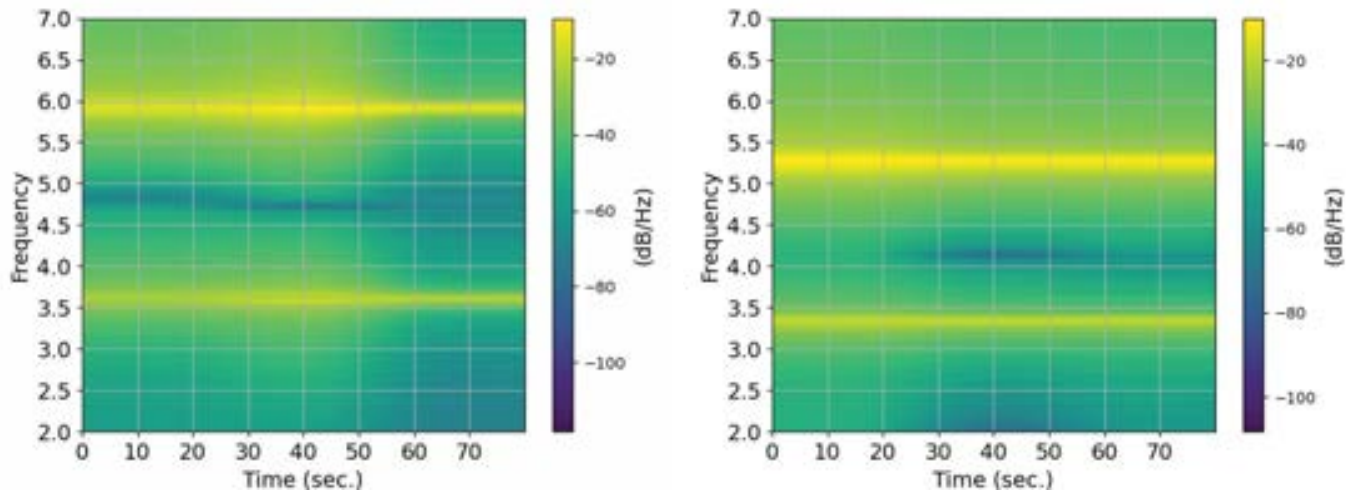

Figure 8: Natural frequency spectrum. Left: Level 1. Right: Level 7.

\subsection{Influence of temperature and prestress change}

On the measurement day, the ambient temperature increased from $28{ }^{\circ} \mathrm{C}$ to $30^{\circ} \mathrm{C}$ during the prestressing adjustment from Level 1 to Level 9. Therefore, the high prestress level coincides with the high measured concrete temperature. The increasement in concrete temperature was higher than that of the ambient temperature. The results (Fig. 9 and 10) show that in our case the velocity variation is independent from the temperature change.
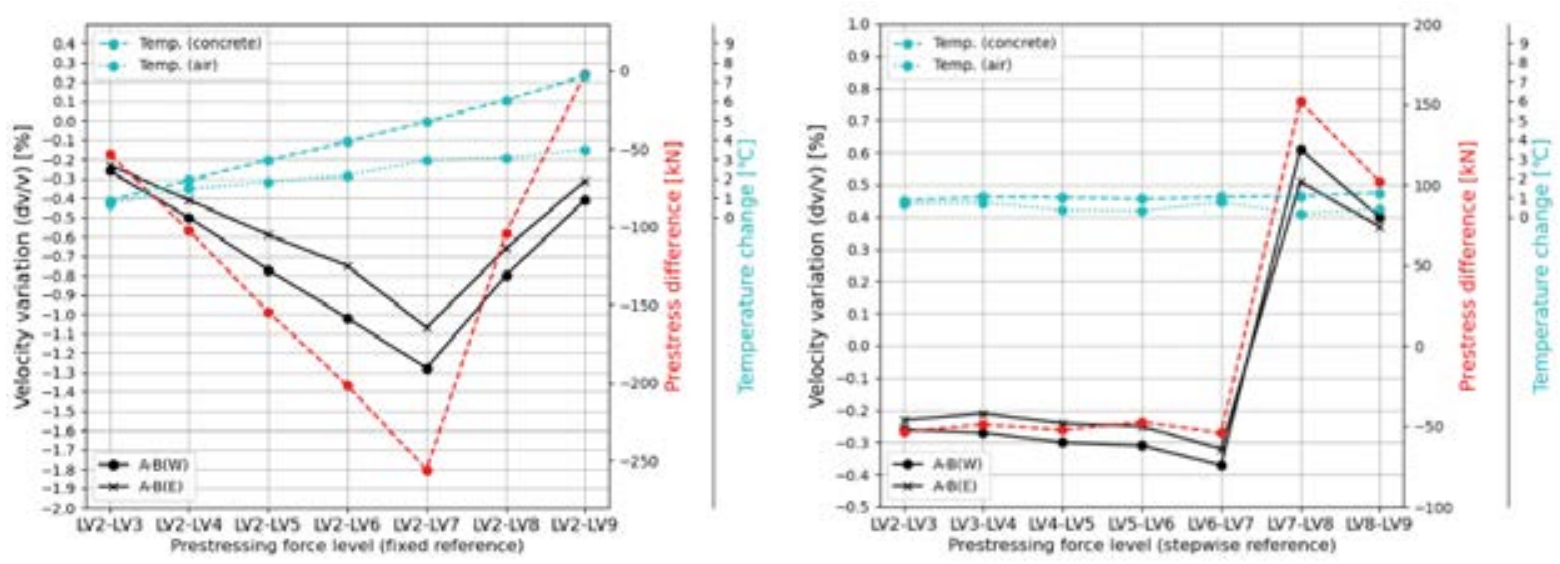

Figure 9: Ultrasonic coda velocity variation. Left: the fixed reference (Level 2). Right: the stepwise reference.
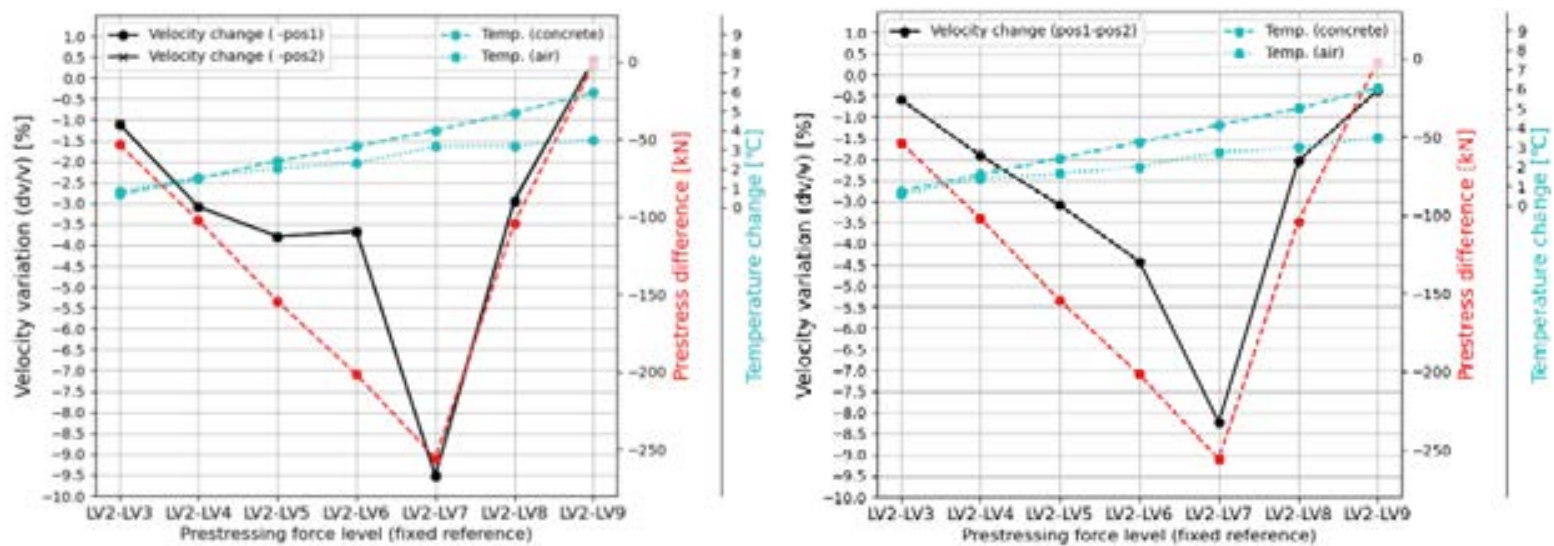

Figure 10: Impulse wave velocity variation (fixed reference: Level 2). Left: obtained from the real impulse response. Right: obtained from the virtual impulse response. 


\subsection{Discussion}

Although Fig. 8 shows the natural frequency change due to the overall stiffness change, it is difficult to quantify the real damage condition and even more difficult to detect the structural property alteration with smaller prestress changes. Moreover, the natural frequency as a global dynamic characteristic is not sensitive to the damage location.

Fig. 9 and 10 show that the variation in velocity for both the ultrasonic coda and the impulse response coda corresponds very well with the prestress change. Particularly the velocity variation of the ultrasonic coda was greater in the west side than in the east sides (Fig. 9 left). The high sensitivity of the ultrasonic coda came as a benefit in the detection of the local damage condition. The wave velocity variations in the impulse response received at the position 1 and 2 were the same (Fig. 10 left). Thus, the hammer impact had the same influence on both receiver positions. Comparing the results shown in Fig. 10, the velocity variation in the virtual impulse response fits the prestress change better than the real impulse response. The applicability of seismic interferometry used to extract the virtual impulse response for CWI is approved. In addition, a significant drop in velocity variation was observed between Level 6 and 7. We state that this result is caused by the open cracks. This revealed the influence of the post-tensioning force on the already existing cracks.

\section{SUMMARY AND OUTLOOK}

Contrary to many researchers who have focused on the assessment of ultrasonic waves in concrete specimens in the laboratory, our study explored the applicability of ultrasound on a large-scale bridge model in a field experiment. The damage condition and the experiment setting made this bridge an ideal test structure to perform not only active, but also passive monitoring. Coda wave interferometry was applied to the ultrasonic coda and the vibration recordings. We investigated the effect of the prestressing force change based on the coda wave velocity variation. The monitoring schemes were verified by the good match of the coda wave velocity variation and the prestress alteration.

One drawback of our experiment is that the ultrasonic measurement duration at different prestress levels is too short to obtain the relationship between the temperature and the wave velocity. Performing a long-term measurement without prestress change will be our next step to investigate the dependence of the coda wave velocity variation on temperature.

\section{ACKNOWLEDGEMENTS}

Thanks to Eva Viefhues, Fred Ziegler and René Seefeld from BAM division 7.2 for their support of our experiment.

This study is part of the GIOTTO project, funded by Bundesministerium für Bildung und Forschung (BMBF) in the frame of "Früherkennung von Erdbeben und ihren Folgen" program (Grant No: 03G0885D).

\section{REFERENCES}

[1] Maeck, J.; Abdel Wahab, M.; De Roeck, G.: Damage Detection in Reinforced Concrete Structures by Dynamic System Identification. In: Proceedings of the International Seminar on Modal Analysis, (2)1999, 939-946. 
[2] Planès, T.; Larose, E.: A Review of Ultrasonic Coda Wave Interferometry in Concrete. In: Cem. Concr, 53 (11)2013, 248-255.

[3] Grêt, A.; Snieder, R.; Scales, J.: Time-lapse Monitoring of Rock Properties with Coda Wave Interferometry. In: Journal of Geophysical Research: Solid Earth, 111(B3)2006.

[4] Bassil, A.; Wang, X.; Chapeleau, X.; Niederleithinger, E.; Abraham, O.; Leduc, D.: Distributed Fiber Optics Sensing and Coda Wave Interferometry Techniques for Damage Monitoring in Concrete Structures. In: Sensors, 19(2)2019, 356.

[5] Niederleithinger, E.; Wang, X.; Herbrand, M.; Müller, M.: Processing Ultrasonic Data by Coda Wave Interferometry to Monitor Load Tests of Concrete Beams. In: Sensors, 18(6)2018, 1971.

[6] Salvermoser, J.; Hadziioannou, C.; Stähler, S. C.: Structural Monitoring of a Highway Bridge Using Passive Noise Recordings from Street Traffic. In: The Journal of the Acoustical Society of America, 138(6)2015, 3864-3872.

[7] Wang, X.; Chakraborty, J.; Bassil, A.; Niederleithinger, E.: Detection of Multiple Cracks in Four-Point Bending Tests Using the Coda Wave Interferometry Method. In: Sensors, 20(7)2020, 1986. https://doi.org/10.3390/s20071986

[8] Unger, J. F., Gründer, K.-P., Hille, F., Liehr, S., Maack, S., Niederleithinger, E., Pirskawetz, S., Rogge, A., Said, S., Viefhues, E. et al.: Innovative Messsysteme zum Brückenmonitoring am Beispiel einer Versuchsbrücke - vom Sensor bis zur Zustandsprognose. In: Messen im Bauwesen, 2017, 7-19.

[9] Niederleithinger, E. ; Wolf, J.; Mielentz, F.; Wiggenhauser, H.; Pirskawetz, S.: Embedded Ultrasonic Transducers for Active and Passive Concrete Monitoring. In: Sensors, 15(5)2015, 9756-9772. https://doi.org/10.3390/s150509756. 